\title{
Supervisory Fault Tolerant Control of the GTM UAV Using LPV Methods*
}

\author{
T. Péni ${ }^{1}$, B. Vanek ${ }^{1,2}$, Z. Szabó ${ }^{1}$, P. Gáspár ${ }^{1}$ and J. Bokor ${ }^{1}$
}

\begin{abstract}
The aim of the paper is to present a supervisory decentralized architecture for the design and development of reconfigurable and fault-tolerant control systems in aerial vehicles. The performance specifications with respect to flight envelope and fault states are guaranteed by local controllers, while the coordination of these components is provided by a supervisor. The monitoring components and FDI filters provide the supervisor with information about different fault operations, based on that it is able to make decisions about necessary interventions into the vehicle motions and guarantee reconfigurable and fault-tolerant operation of the aircraft. The design of the proposed reconfigurable and fault-tolerant control is based on an LPV method that uses monitored scheduling variables during the operation of the vehicle. The design is demonstrated on the high-fidelity simulation model of the NASA AirSTAR Flight Test Vehicle.
\end{abstract}

\section{INTRODUCTION}

A major goal in modern flight control system research is the need to pursue improved reliability and environmental sustainability of safety critical systems [14], [13]. Faults and failures may result in loss of performance and even in catastrophic harm or loss of life. For that reason, improving the safety and reliability of commercial aircraft via improving the pilots abilities to counteract the faults and provide them the flight envelope protection functions as long as possible are important priorities. Reconfigurable control methods promise a way to compensate for failures or damage of flight control effectors by using the remaining flight control surfaces to generate compensating forces and moments, via changes in the flight control algorithms. The majority of faults can be detected immediately with system checks, but a few of them requires fault detection and isolation methods (FDI). Combined with reconfigurable control methods they are considered as active fault tolerant control (FTC), which assumes a well defined mechanism to actively detect and counteract against faults in the system [24], [16]. In passive FTC controllers are fixed and are designed to be robust against a class of presumed faults. This approach does not need a fault estimate (or detection) or controller reconfiguration, but provides only limited faulttolerant capabilities [32].

In general, a fault tolerant flight control system is required to perform failure detection, identification and accommodation for sensor and actuator failures. Active FTC schemes can be broadly classified into projection based and online controller redesign based approaches [22]. Numerous results have been proposed related to both approaches in the past few years [9], [1]. More recently in a numerous research projects, within the EU FP7 framework, focus on the environmental impact of the aircraft and hence higher performance is required from the flight control system, with

\footnotetext{
*The research leading to these results has received funding from the European Union Seventh Framework Programme (FP7/2007- 2013) under grant agreement n 2314544. Reconfiguration of Control in Flight for Integral Global Upset Recovery (Reconfigure) project, Andres Marcos coordinator.

${ }^{1}$ the authors are with MTA SZTAKI, Budapest, Hungary

2 corresponding author: vanek at sztaki.hu
}

certifiable algorithms, leading to a paradigm shift from robust passive FTC towards active methods relying on switching, gain scheduled or linear parameter-varying (LPV) methods.

The individual control components used in a vehicle system are often designed decoupled but are in interaction or even conflict with each other in terms of the full vehicle. An integrated control system is designed in such a way that the effects of a control system on other vehicle functions are taken into consideration in the design process by addressing the various performance specifications. Redundancy on sensor and actuator levels makes it possible to realize the same functionality using different sensor and actuator configurations, i.e., control reconfigurations. Thus integrated design is motivated by the needs of reconfigurable and reliable control, see, e.g., [23], [25]. Recently, several important papers have been presented in this topic, see, e.g., [31], [26], [10].

A possible solution to integrated control is to set the design problem for the whole vehicle and include all the performance demands in a single specification. Besides the complexity of the resulting problem the formulation of a suitable performance specification is the main obstacle for this direct global approach. In the framework of available design techniques the formulation and successful solution of complex multi-objective control tasks are highly nontrivial, see, e.g., [12], [3].

Another solution to the integrated control is a quasi decentralized control structure where the components are designed independently, see, e.g., [30], [11]. In the present paper the quasi decentralized control system is augmented with a supervisor as illustrated in Figure 1. The role of the supervisor is to meet performance specification in different operating modes, i.e. fault conditions, and prevent the interference between flight control components. The supervisor has information about the current operational mode of the vehicle, i.e., the various vehicle maneuvers or the different fault operations gathered from monitoring components and fault-detection and identification (FDI) filters. The supervisor is able to make decisions about the necessary interventions into the vehicle components and guarantee the reconfigurable and fault-tolerant operation of the vehicle. These decisions are propagated to the lower layers through predefined interfaces encoded as suitable scheduling signals using the LPV framework.

The present paper proposes a reconfiguration approach for longitudinal motion of a sub scale aircraft based on the a priori synthesis of a Linear Parameter Varying (LPV) controller, which can be further extended to lateral control with more performance specifications which can be effectively handled by a lower complexity supervisor. The fault signal generated by an FDI algorithm is formulated as a parameter on which the LPV controller is scheduled. The prime advantage of this approach is that the synthesis results in a single multivariable controller with stability and robustness guarantees for the closed-loop system. The paper is organized as follows. Section II presents a brief introduction to the supervisory architecture. The vehicle model of the NASA AIRStar UAV 
are presented in Section III. In Section IV the considerations related to controller synthesis are discussed followed by simulation results in Section V. Some conclusions are drawn in Section VI.

\section{ARCHitecture}

The term configuration refers to a well-defined sensor and actuator set that is associated with a given functionality. Control reconfiguration is motivated by the following requirements: the achieved control performance in certain scenarios must be improved and an increased reliability in the presence of sensor or actuator faults must be achieved. The term event is related to the occurrence of such a scenario. A finite set of events $\mathcal{E}$ are always assumed. On a higher level an event is handled based on a given functionality thus one can associate a certain event $e \in \mathcal{E}$ with a set of configurations $\mathcal{C}_{e}$. Reconfigurable control strategies define a policy $\mathcal{S}: \mathcal{E} \times \mathcal{C} \rightarrow$ $\mathcal{C}_{e}$ to select a possibly new configuration $K=\mathcal{S}(e)$ when an event $e$ occurs in a specific, usually baseline, configuration. In a normal situation a baseline configuration is handled by a single local component, e.g., elevator for pitch control, otherwise it is composed of several local components that can fulfill the same functional behavior, e.g., elevator, engines and spoilers for generating pitch moment. The event set $\mathcal{E}$, the corresponding class of the configuration sets $\mathcal{C}_{e}$ and the policy $\mathcal{S}$ are determined in the preliminary step of the design. The specification of these elements may be a highly nontrivial task when considering the high number of flight control surfaces and sensors onboard the aircraft.

\section{A. Supervisor}

The role of the supervisor is to coordinate the local components and handle the interactions between them. Since the performance specifications of local controllers are often in conflict, the supervisor must also guarantee a balance or trade-off between them. This trade-off is formulated on the level of local controllers as a result of engineering knowledge. However, when an event occurs the preferences. i.e., the trade-off levels, are subject to change. This fact is reflected in the structure of the policy $\mathcal{S}$. As an example, the effect of actuator saturation may prevent a specific performance requirement to be fulfilled. The encountered performance degradation might be treated as an event, if there is a configuration that potentially can improve the performance, which in practical situations is closely related to a safety requirement. Recovering to the normal parameters creates another event that makes the supervisor to select the baseline configuration.

In order to implement a transition defined by the policy $\mathcal{S}$, a safety feature, the operation of a local controller must be modified by a supervisory command. This is realized through a set of scheduling variables that are transmitted to the local controllers. At a local level the behavior of the controller is affected by these scheduling variables through the performance weighting functions. The difficulty in the supervisory control is that global stability and performance are difficult to guarantee. The information provided by the supervisor is composed of messages and signals sent by the monitoring components and fault detection and isolation (FDI) filters. Based on this information the supervisor is able to make decisions about the necessary vehicle maneuvers and guarantee reconfigurable and fault-tolerant operation of the vehicle and send messages to the local controllers.

The design of the supervisor does not involve dynamical systems explicitly. However, due to the time variation of the signals the designer should check the validity of relations

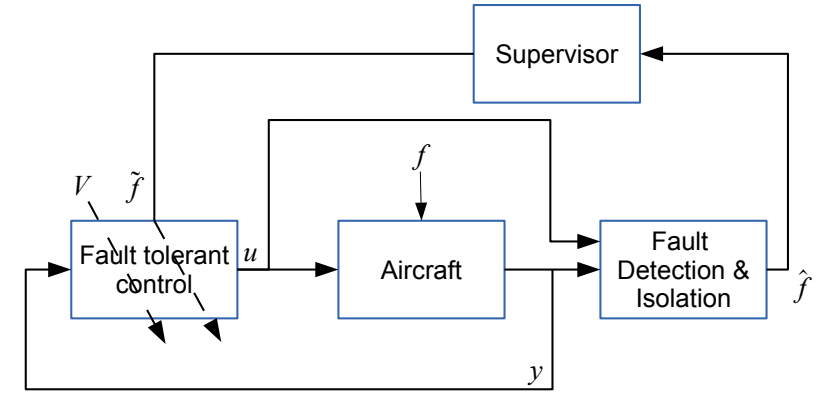

Fig. 1. Schematics of the supervisory framework

between the momentary values of the monitoring signals based on a temporal logic. The difficult part of the design is to ensure the correctness of the specification, see [21], [19]. It must be stressed at this point that the baseline configurations handle only a subset of actuators, associated with a given task (functionality). The hierarchy of the configurations and corresponding scheduling variables ensure that the additional actuator(s) considered improve the stability properties of the given functionality.

In contrast to the controller switching strategy the proposed approach uses a performance weighting strategy. On the supervisor level the required configurations are defined uniquely by the specific values of a set of marker signals. These marker signals are used as scheduling variables on the level of local controllers. The task of the supervisor design is to specify these marker signals in such a way that the different combinations of their values define the specific event (functionality) in a unique way. The different combinations of the marker signals encode the designers specification (option) in dealing with multi-objective or conflicting scenarios.

As an illustration consider the altitude tracking problem which is handled by using elevator. When the vehicle is performing a maneuver, e.g., a flight level change or turning, the change in angle-of-attack (AoA) might generate increased drag. The role of the engine is to provide thrust and keep the vehicle at constant speed. However, using the engine with slow dynamics and the elevator with fast response might lead to stall. Hence, to balance the contradicting requirements of altitude change and AoA limitation an active supervisor has to handle reference commands and might have to switch between throttle and elevator used for tracking commands. The current paper considers a supervisory framework with an aircraft longitudinal motion, where in normal mode the pitch rate commands are followed by using elevator deflections, but in case a fault occurs the system is reconfigured to use spoilers and engines with the remaining authority of the elevator, as shown in Figure 1.

\section{B. Local LPV Control}

LPV techniques offer a systematic design methodology to address control of highly coupled, nonlinear uncertain dynamic systems. The benefits of this approach include guaranteed global stability and robust performance, and realtime implementation of these controllers [29]. Linear Parameter Varying control uses the $\mathcal{H}_{\infty}$-robust control framework. Where frequency dependent weighting functions are used to describe the required properties of the controller. In the same manner as in linear time invariant case, the controller synthesis has a weighted interconnection setup, where design 
weight are used to form the performance specifications. The plant and the resulting controller are both parameter depending and to cast into an optimization the parameter dependent closed-loop transmission is minimized. The induced $\mathcal{L}_{2}$ norm ([29]) can be minimized by a parameter varying controller in the form:

$$
\left[\begin{array}{c}
\dot{x}_{c}(t) \\
u(t)
\end{array}\right]=\left[\begin{array}{ll}
A_{c}(\rho(t)) & B_{c}(\rho(t)) \\
C_{c}(\rho(t)) & D_{c}(\rho(t))
\end{array}\right]\left[\begin{array}{c}
x_{c}(t) \\
y(t)
\end{array}\right],
$$

where $\rho \in \mathcal{F}_{\mathcal{P}}$ can be measured in realtime. By the appropriate selection of weights in the generalized interconnection ( Fig. 2) the closed loop system $G$ can be chosen such that the induced $\mathcal{L}_{2}$ norm of the system can not overpass a certain level, denoted by $\gamma$.

The controller synthesis problem can be cast into a series of Linear Matrix Inequalities (LMIs). More interested reader is referred to [15], where the method is described in great details.

The real time application of the LPV controller requires, first, the off-line computation of the controller and $\rho$ has to be available in real time for scheduling. Therefore, this approach allows to construct an LPV controller whose dynamics adjust according to variations in plant dynamics, such as mass, velocity, aerodynamic parameters, or fault states and maintain prescribed stability and performance along all parameter trajectories.

\section{Fault Detection}

In analytical redundancy schemes, the resulting difference generated from the consistency checking of different variables is called as a residual signal. The residual should be zero when the system is normal, and should diverge from zero when a fault occurs in the system. This zero and non-zero property of the residual is used to determine whether or not faults have occurred. Analytical redundancy makes use of a mathematical model and the goal is the determination of faults of a system from the comparison of available system measurements with a priori information represented by the mathematical model, through generation of residual quantities and their analysis. Various approaches have been applied to the residual generation problem, the parity space approach [4], [20], [17], [28]. Most of these design approaches refer to linear time-invariant (LTI) systems. The geometric concept is further generalized to linear parameter-varying (LPV) systems by [2], while input affine nonlinear systems are considered by [6]. The basic concepts underlying observer-based fault detection and isolation (FDI) schemes are the generation of residuals and the use of an optimal or adaptive threshold function to differentiate faults from disturbances, see the surveys of [8], [20]. The threshold function is used to robustify the detection of the fault by minimizing the effects from false faults, disturbances and commands on the residuals. For fault isolation, the generated residual has to include enough information to differentiate said fault from another, usually this is accomplished through structured residuals or directional vectors. Robustness of the FDI algorithm is determined by its capability to decouple the filter performance outputs from disturbances, errors, and unmodelled dynamics. In the present paper the fault detection filter is not designed, only a simplified representation is adopted based on the results from elevator FDI on commercial aircraft [27]. It has an approximate dynamics of $G_{F D I}=\frac{5.129}{s+5.129}$, which estimates the loss of efficiency of the elevator surface. Assuming a jamming fault on one of the two elevators, FDI output reaches an expected value of 0.5 with some time lag, resulting in model discrepancy during

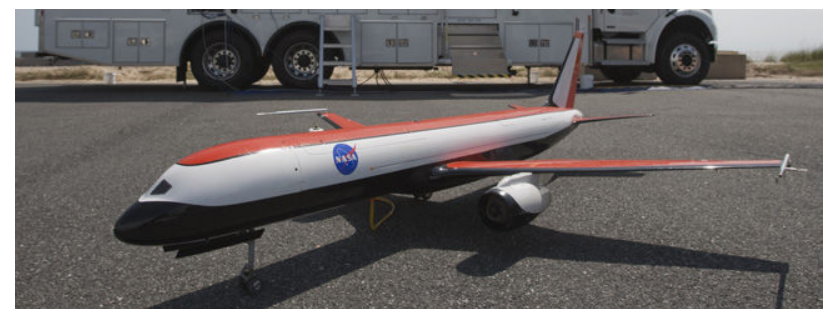

Fig. 3. The NASA AIRStar vehicle.

the fault detection transient, leading to mismatch between the true and estimated LPV fault scheduling variable.

\section{VEHICLE MODEL}

An effective resource for experimentally testing fight control algorithms, including adaptive control algorithms, is the Airborne Subscale Transport Aircraft Research (AirSTAR) testbed at NASA Langley Research Center [18]. The primary AirSTAR flight test vehicle is a turbine powered $5.5 \%$ dynamically scaled model of a civilian transport aircraft, often referred to as the Generic Transport Model (GTM). The GTM has a wing span of $7 \mathrm{ft}$, and weighs around $55 \mathrm{lbs}$. Under normal operations, it flies at an altitude of 700 to $1100 \mathrm{ft}$, with an airspeed between 70 and 85 knots. The currently used T-2 test aircraft is shown in Figure 3. Significant wind tunnel and flight testing has been performed to identify the flight dynamics of the GTM [5]. A nonlinear simulation model of the aircraft dynamics has been developed and is readily available to the research community. Experimental control algorithms are easily embedded in this simulation model for verification prior to flight testing [7]. Hence, the AirSTAR testbed is a highly effective for experimental flight control research through its flexible architecture and rapid implementation and testing cycle. GTM nonlinear simulation model is trimmed to steady level flight at 60 to 100 knots at 10 knots interval and linearized to obtain a set of linear models of the aircraft dynamics. A twelve state, full-order linear model is generated for the given flight condition. The four states governing longitude, latitude, altitude, and heading are eliminated as they have no impact on the flight dynamics. The remaining eight states, reduced-order model fully describes the longitudinal, lateral, and directional dynamics of the aircraft. Coupling between the longitudinal and lateral/directional axes is limited, and can be neglected for the purpose of control design. Hence, the longitudinal controller described here is designed independently of the lateral/directional controller.

A four state model, decoupled from the eight state reduced-order model, captures the primary longitudinal flight dynamics of the GTM. The states of the model are forward airspeed $u[\mathrm{~m} / \mathrm{s}]$, vertical speed $w[\mathrm{rad}]$, pitch rate $q[\mathrm{rad} / \mathrm{s}]$, and pitch angle $[\mathrm{rad}]$. The control input to the model are left and right elevator deflection $\delta_{e, L}, \delta_{e, R}[\mathrm{rad}]$, spoiler deflection $\delta_{s p}[\mathrm{rad}]$, and throttle $\delta_{T H R}[\%]$. The longitudinal LPV state-space model of the GTM between 60 and 100 knots is approximated with an affine LPV model in the form of:

$$
\left[\begin{array}{c}
\dot{q} \\
\dot{u} \\
\dot{w} \\
\dot{\theta}
\end{array}\right]=\left(A_{0}+A_{V} V_{\text {cas }}\right)\left[\begin{array}{c}
q \\
u \\
w \\
\theta
\end{array}\right]+\left(B_{0}+B_{V} V_{\text {cas }}\right)\left[\begin{array}{c}
\delta_{e, L} \\
\delta_{e, R} \\
\delta_{s p} \\
\delta_{T H R}
\end{array}\right]
$$




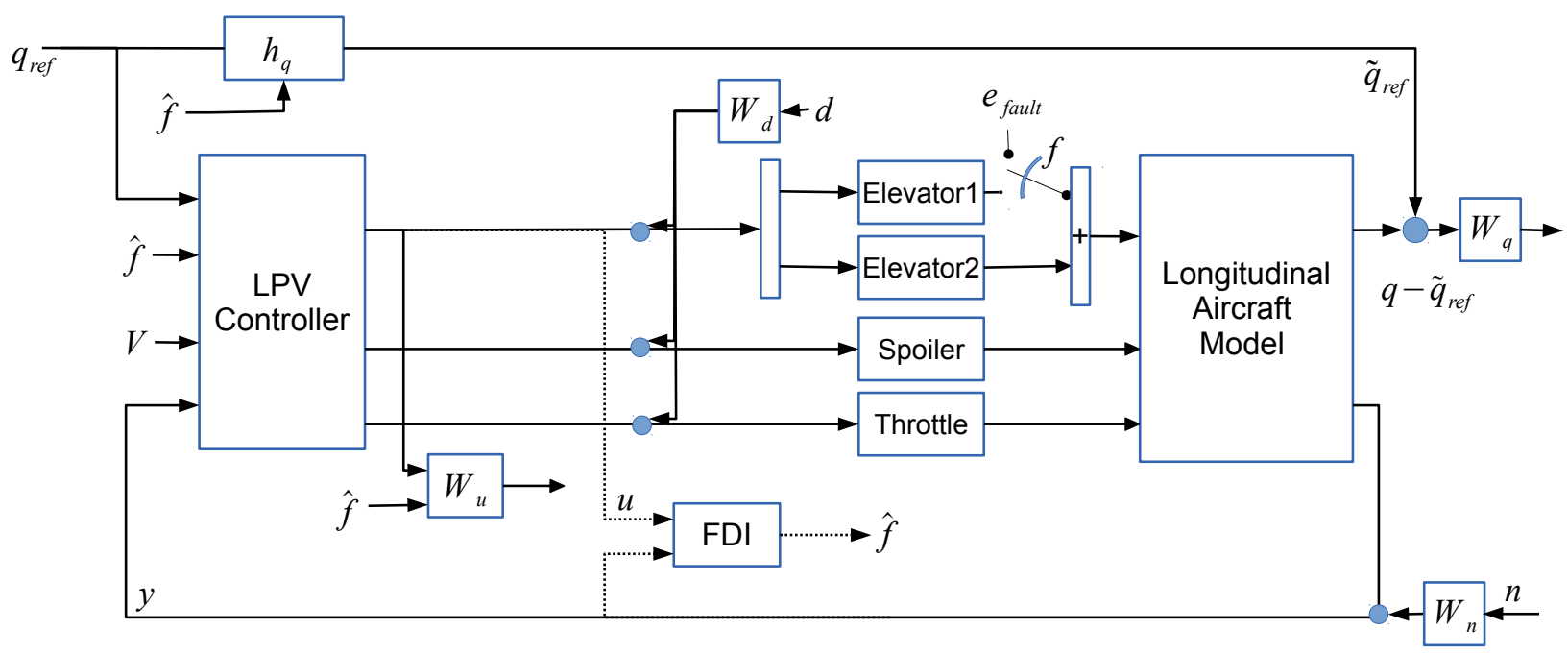

Fig. 2. Weighted interconnection of the control problem

with outputs of pitch rate and normal acceleration

$$
\left[\begin{array}{c}
q \\
a_{z}
\end{array}\right]=\left(C_{0}+C_{V} V_{c a s}\right)\left[\begin{array}{c}
q \\
u \\
w \\
\theta
\end{array}\right]+\left(D_{0}+D_{V} V_{c a s}\right)\left[\begin{array}{c}
\delta_{e, L} \\
\delta_{e, R} \\
\delta_{s p} \\
\delta_{T H R}
\end{array}\right]
$$

where the affine LPV coefficients are obtained with least square fit on the pointwise LTI plants. The plant is augmented with first order actuator dynamics of $G_{a c t}=\frac{5}{s+5}$ on elevator and spoiler and $G_{e n g}=\frac{0.1474 s+0.7314}{s^{2}+1.336 s+0.7314}$ on the the throttle, which contains a right half plane zero. Sensor dynamics are omitted due to the high quality of the sensors onboard. The second scheduling parameter is the right elevator fault, which results in a zero second column in the corresponding $B$ and $D$ matrices in the case of complete jamming of the elevator.

\section{Control Design}

The system interconnection (Fig. 2) addressing the robust parameter dependent controller synthesis proposed to solve the pitch rate tracking problem assuming model mismatches and exogenous disturbance is detailed in the following. The influence of actuator input uncertainty is denoted with $W_{d}$ with weight of

$$
W_{d}=<\frac{2 s+8}{s+160} ; \frac{3 s+12}{s+160} ; \frac{1.5 s+30}{s+200}>
$$

$<>$ representing a diagonal matrix, with the least uncertainty on elevator, higher uncertainty on spoiler channel, while the amount of uncertainty at higher frequency on the throttle is lower, due to the limited bandwidth of this actuator.

Actuator usage is penalized in the design with weights of $W_{u}$, where the design weights are parameter dependent, scheduled with the fault magnitude, in nominal case $W_{u}^{n}=<$ $1 / 30 ; 1 / 5 ; 1 / 5>$ corresponding to the allowed magnitude of deflection in the robust control synthesis, due to loss of elevator in the faulty case spoiler and throttle can be used more actively with weights of $W_{u}^{f}=<1 / 20 ; 1 / 15 ; 1 / 10>$ leading to higher spoiler deflections and more throttle usage.

Characteristics of the noise is captured by $W_{n}=<$ $\frac{0.25 s+0.25}{s+100} ; \frac{0.05 s+0.05}{s+100}>$ with weights on $q, a_{z}$ with fre- quency dependent magnitude and higher noise on pitch rate sensor due to the dimension used.

Model matching is achieved by filtering the reference signal through a "handling-qualities" model $\left(h_{q}\right)$ to achieve smooth behavior with adequate speed of response for pitch rate commands. The model represents the ideal behavior of the vehicle for pilot inputs, it has high speed of response in nominal case $W_{r e f}^{n}=\frac{7.5^{2}}{s^{2}+15 s+7.5^{2}}$ and have slower reference behavior in the faulty case $W_{r e f}^{n}=\frac{4^{2}}{s^{2}+8 s+4^{2}}$ depending on the fault scheduling parameter.

The main control objective, to keep the error between the plant output $q$ and the desired response $h_{q}$ low, is weighted across frequency with $W_{q}=100 \frac{15^{2}}{s^{2}+30 s+15^{2}}$ across all parameter range, trading off good steady state tracking with degraded performance at frequencies higher than $15 \mathrm{rad} / \mathrm{s}$.

The weights are optimized with linear point design first, at 10 points of the parameter space $\rho_{1}=[60: 10: 100] ; \rho_{2}=$ $[0 ; 0.5]$ in the LPV model. The present method takes advantage of parameter dependent performance weights, which significantly improves the tradeoff between higher tracking performance at healthy operating state and reduce the speed of tracking response with higher actuator load at faulty operation.

During the pointwise $\mathcal{H}_{\infty}$ synthesis the $\gamma$ performance level ranges between 1.68 and 1.83. While the LPV synthesis with unbounded parameter rate, with constant Lyapunov function leads to a higher $\gamma$ performance level, the $\mathcal{L}_{2}$ gain is 1.98. This is a consequence of using a single, parameter dependent LPV controller, where the parameter rates can be unbounded.

\section{Simulation}

The performance of the obtained longitudinal control system is assessed in a nonlinear simulation environment of the AirSTAR vehicle. To provide sufficient evidence of the applicability of the present method, the aircraft is released from a non trimmed flight condition, with significant variation in the calibrated airspeed $V_{\text {cas }}$, as shown in Figure 4 , which is the scheduling parameter of the plant. 


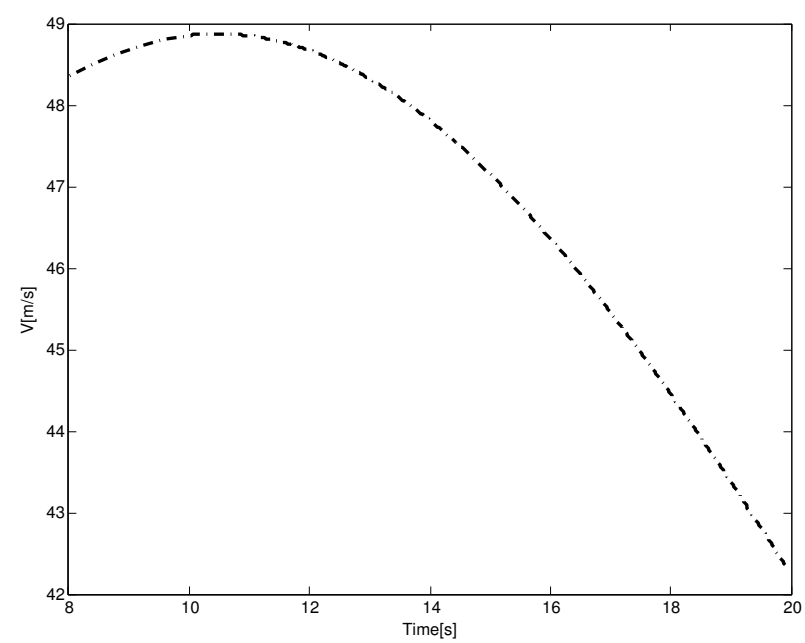

Fig. 4. Parameter trajectory of the scheduling variable $V_{\text {cas }}$.

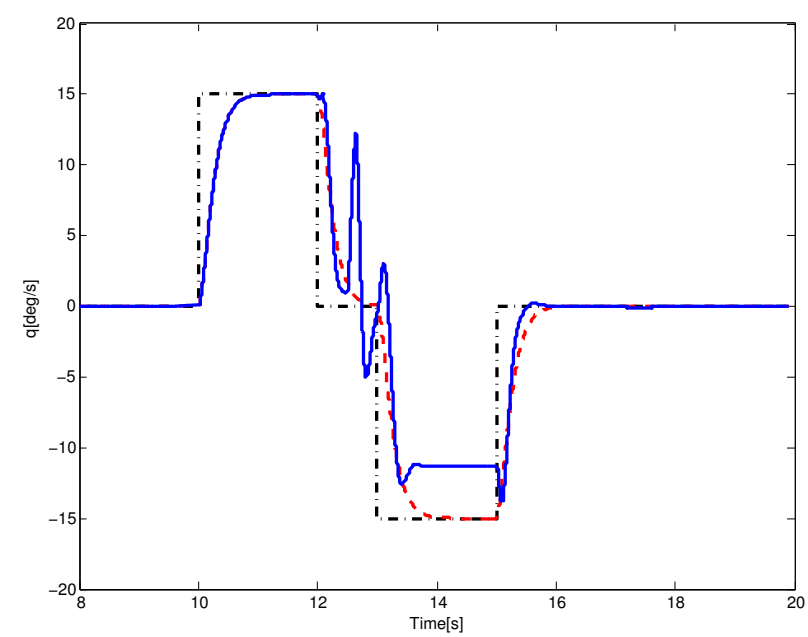

Fig. 5. Baseline LPV controller without fault scheduling, tracking response with fault occurring at $12.5 \mathrm{~s}$.

The baseline flight control problem is to follow a $15 \mathrm{deg} / \mathrm{s}$ pitch rate doublet, with the specified handling quality model. Which is achieved almost perfectly in the fault free case, as shown in the first $12.5 \mathrm{~s}$ of Figure 5. But as the elevator jamming fault occurs at $12.5 \mathrm{~s}$, the tracking performance degrades unacceptably.

The corresponding control inputs on elevator, spoiler and throttle are also becoming unacceptable, as shown in Figure 6 , note that actuator limits are enforced on all channels, corresponding to $\pm 30 \mathrm{deg}$ on elevator, $0-30 \mathrm{deg}$ on spoiler, and $0-100 \%$ on the throttle. As described above the FDI filter is assumed to follow a dynamic behavior to estimate the fault magnitude, as shown in Figure 7. Which gives a reasonable approximation of the behavior of the fault detection transient when a sudden jamming on the right elevator occurs. In case the LPV controller is scheduled with the fault estimate, besides the calibrated airspeed, the tracking response is significantly improved as shown in Figure 8. It is clearly visible, that the ideal reference behavior is significantly slower but the aircraft is able to execute the maneuver without steady-state error and unwanted oscillations.
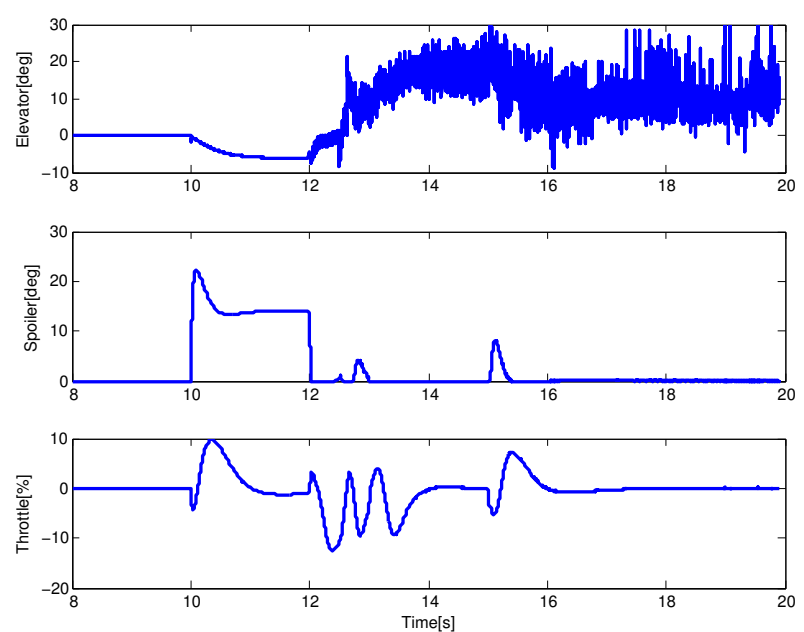

Fig. 6. Baseline control signals with fault occurring at $12.5 \mathrm{~s}$.

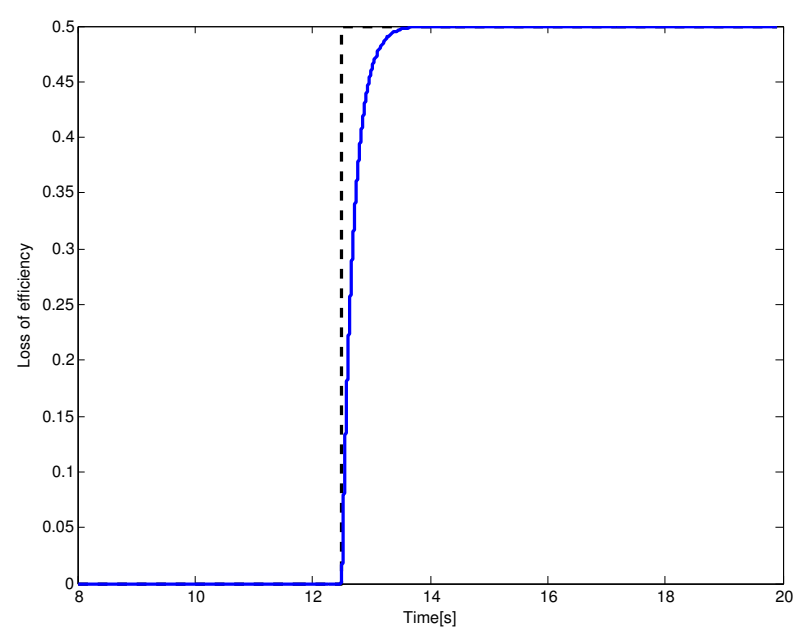

Fig. 7. The FDI output detecting the right elevator fault, modeled as loss of efficiency.

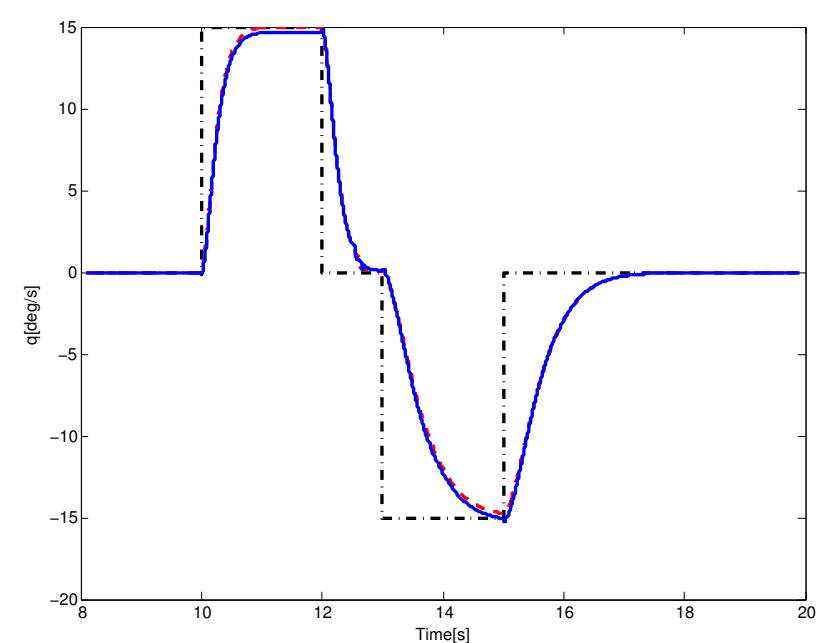

Fig. 8. Reconfigurable LPV controller with fault scheduling, tracking response with fault occurring at $12.5 \mathrm{~s}$. 

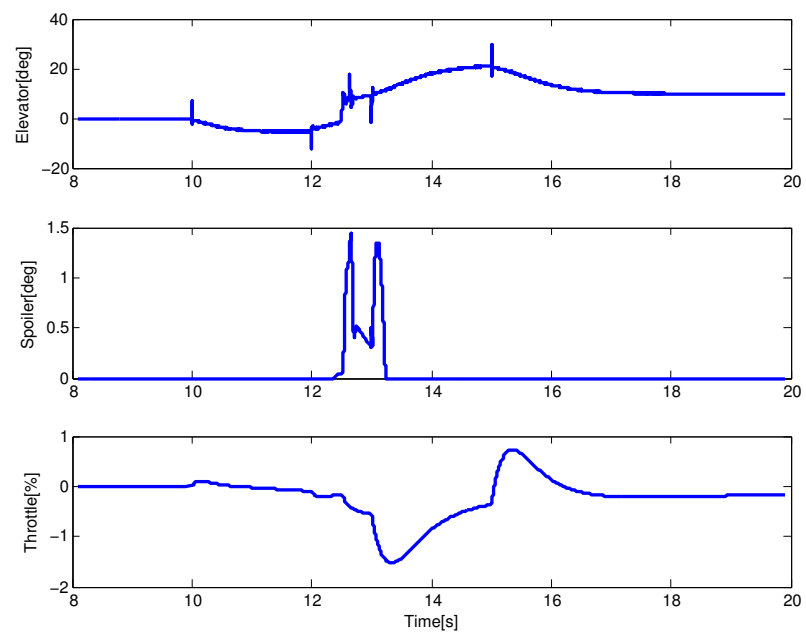

Fig. 9. Reconfigurable LPV control signals with fault occurring at $12.5 \mathrm{~s}$.

The control inputs during the initial phase of the maneuver are respecting the design goals, of using mainly the elevator actuator, while after suffering from the loss of one elevator the supervisor is reallocating the resources, scheduling with the faults estimate, to use spoilers and engine also for pitch rate tracking, as shown in Figure 9.

The advantage of the reconfigurable approach is apparent from the results presented above, and the design framework can be extended to lateral, longitudinal and altitude control with a more complex supervisory logic, where the performance tradeoffs are not only optimized for one single fault condition but several of them, in addition to the turn coordination tasks where usage of control inputs creates cross coupling.

\section{CONCLUSION}

The paper presents a longitudinal example of a supervisory framework for fault tolerant reconfigurable flight control. The presented method uses the LPV control design framework, where flight envelope parameters and fault states of the system can be used as scheduling variables. The corresponding LPV design setup uses fault parameter dependent performance weights to provide good tradeoff between high performance operation in nominal and robust lower performance operation in degraded mode. The present framework, with a simple supervisor serves as a baseline for the full aircraft control problem with lateral and longitudinal control, coupled via the supervisor capable of addressing several actuator and sensor fault cases. The applicability of the method is validated on the high-fidelity aircraft model of the NASA AirSTAR.

\section{REFERENCES}

[1] Halim Alwi, Christopher Edwards, and CheePin Tan. Fault Detection and Fault-Tolerant Control Using Sliding Modes. Springer-Verlag, 2011.

[2] G. Balas, J. Bokor, and Z. Szabo. Invariant subspaces for LPV systems and their applications. IEEE Transactions on Automatic Control, 48(11):2065-2069, 2003.

[3] G. Burgio and P. Zegelaar. Integrated vehicle control using steering and brakes. International Journal of Control, 79:534-541, 2006.

[4] E.Y. Chow and A.S. Willsky. Analytical redundancy and the design of robust failure detection systems. IEEE Trans. on Automatic Control, 29(7):603-614, 1984.

[5] K. Cunningham, J. V. Foster, A. M. Murch, and E. Morelli. Practical application of a subscale transport aircraft for flight research in control upset and failure conditions. In AIAA Guidance, Navigation, and Control Conference, 2008.
[6] C. De Persis, R. De Santis, and A. Isidori. Nonlinear actuator fault detection and isolation for a VTOL aircraft. In Proceedings of the 2001 American Control Conference, Vols 1-6, pages 4449-4454, 2001.

[7] A. Dorobantu, A. M. Murch, and G. J. Balas. $h_{\infty}$ robust control design for the nasa airstar flight test vehicle. In 50th AIAA Aerospace Sciences Meeting, 2012.

[8] P.M Frank. Fault diagnosis in dynamic systems using analytical and knowledge-based redundancy - a survey and some new results. Automatica, 26:459-474, 1990.

[9] S. Ganguli, A. Marcos, and Gary Balas. Reconfigurable lpv control design for boeing 747-100/200 longitudinal axis. In American Control Conference, 2002. Proceedings of the 2002, volume 5, pages 36123617 vol.5, 2002.

[10] P. Gaspar, B. Nemeth, and J. Bokor. Design of an lpv-based integrated control for driver assistance systems. In Robust Control Design (7th ROCOND), 2012

[11] P. Gaspar, Z. Szabo, and J. Bokor. An integrated vehicle control with actuator reconfiguration. IFAC World Congress, Seoul, Korea, 2008.

[12] T. Gordon, M. Howell, and F. Brandao. Integrated control methodologies for road vehicles. Vehicle System Dynamics, 40:157-190, 2003.

[13] Philippe Goupil and Andres Marcos. Industrial benchmarking and evaluation of addsafe fdd designs. In Fault Detection, Supervision and Safety of Technical Processes (8th SAFEPROCESS), 2012.

[14] Jens Knig and Thomas Hellstrom. The clean sky smart fixed wing aircraft integrated technology demonstrator: Technology targets and project status. In 27TH INTERNATIONAL CONGRESS OF THE AERONAUTICAL SCIENCES, 2010.

[15] Lawton H. Lee. Identification and Robust Control of Linear Parameter-Varying Systems. $\mathrm{PhD}$ thesis, University of California Berkeley, 1997

[16] M. Mahmoud, J. Jiang, and Y. Zhang. Active Fault Tolerant Control System. Springer-Verlag, 2003.

[17] M.A. Massoumnia. A geometric approach to the synthesys of failure detection filters. IEEE Transactions on Automatic Control, 31:839_ 846, 1986.

[18] A. M. Murch. A flight control system architecture for the nasa airstar flight test infrastructure. In AIAA Guid ance, Navigation, and Control Conference, 2008.

[19] T. Niinomi, B.H. Krogh, and J.E.R. Cury. Synthesis of supervisory controllers for hybrid systems based on approximating automata. Proc. 34th IEEE Conference on Decision and Control, pages 1461 - 1466, 1995

[20] R. J. Patton and J. Chen. Robust fault detection and isolation FDI systems. Contr. Dynamic Syst., 74:176-224, 1996.

[21] A. Platzer. Differential dynamic logic for verifying parametric hybrid systems. J Autom Reasoning, 41:143-189, 2008.

[22] C. Sloth, T. Esbensen, and J. Stoustrup. Active and passive faulttolerant lpv control of wind turbines. In American Control Conference (ACC), 2010, pages 4640-4646, 2010.

[23] M. Staroswiecki. Robust fault tolerant linear quadratic control based on admissible model matching. 45th IEEE Conference on Decision and Control, pages 3506 - 3511, 2006.

[24] Marc Steinberg. A historical overview of research in reconfigurable flight control. Aerospace Control and Guidance Systems Committee Meeting No. 95, Subcomitee E:1-38, 2005.

[25] J. Stoustrup. Plug and play control: Control technology towards new challenges. European Journal of Control, 15(3-4):311-330, 2009

[26] A. Trachtler. Integrated vehicle dynamics control using active brake, steering and suspension systems. International Journal of Vehicle Design, 36:1-12, 2004

[27] B. Vanek, Z. Szabó, A. Edelmayer, and J. Bokor. Fault detection of electrical flight control system actuators using parameter dependent estimation. In Fault Detection, Supervision and Safety of Technical Processes (IFAC SAFEPROCESS), 2012.

[28] A. Varga. On computing least order fault detectors using rational nullspace bases. In In Proceedings of the IFAC Symp. SAFEPROCESS'2003, Washington D.C., 2003.

[29] F. Wu. Control of linear parameter varying systems. PhD Thesis, Mechanical Engineering, University of California at Berkeley, 1995.

[30] H.S. Xiao, W.W. Chen, H.H. Zhou, and J.W. Zu. Integrated control of active suspension system and electronic stability programme using hierarchical control strategy: theory and experiment. Vehicle System Dynamics, 49:381-397, 2011

[31] F. Yu, D.F. Li, and D.A. Crolla. Integrated vehicle dynamics control: State-of-the art review. IEEE Vehicle Power and Propulsion Conference, Harbin, China, 2008.

[32] Y. Zhang and J. Jiang. Bibliographical review on reconfigurable faulttolerant control systems. Annual Reviews in Control, 32:229-252, 2008 